\title{
The Effect of Participation in a Game of Tag, an Activity Promoting Helping Behavior, on Children's Helping Behavior-Related Self-Efficacy
}

\author{
Kohei Ueno \\ Faculty of Education, Kagawa University, Takamatsu, Japan \\ Email: ueno@ed.kagawa-u.ac.jp
}

How to cite this paper: Ueno, K. (2018). The Effect of Participation in a Game of Tag, an Activity Promoting Helping Behavior, on Children's Helping Behavior-Related Self-Efficacy. Advances in Physical Education, 8, 238-245.

https://doi.org/10.4236/ape.2018.82021

Received: April 17, 2018

Accepted: May 22, 2018

Published: May 25, 2018

Copyright $\odot 2018$ by author and Scientific Research Publishing Inc. This work is licensed under the Creative Commons Attribution International License (CC BY 4.0).

http://creativecommons.org/licenses/by/4.0/

(c) (i) Open Access

\begin{abstract}
With a breakdown in classroom discipline, bullying and social withdrawal have become social problems in recent years; there is a heightened expectation toward physical education for developing proper social attitudes and cultivating morality. In this study, children were instructed to play "Nakama-oni (helping tag)", a tag game variant promoting helping behavior through physical education. Thereafter, the frequency of helping behavior in Nakama-oni was checked, and the effect of participation in physical education through Nakama-oni on children's helping behavior-related self-efficacy was discussed. Sixty elementary school children in the fifth grade played both Nakama-oni and normal tag. The results suggested that: 1) children experienced helping behavior more often in Nakama-oni, compared to normal tag; and 2) participation in Nakama-oni enhanced children's helping behavior-related self-efficacy. In conclusion, results suggest that incorporating Nakama-oni that promotes helping behavior in physical education enhances children's helping behavior-related self-efficacy.
\end{abstract}

\section{Keywords}

Helping Behavior, Tag, Self-Efficacy, Prosocial Behaviors, Empathy

\section{Introduction}

Generalization of the fair play spirit attained through physical activities in daily living is one of the desiderata that modern society expects of physical education. For example, behaviors exhibited in "following rules", "being prepared to do one's best" as well as attitudes toward playing fairly or to care for opponents are 
expected to be reflected in daily life. However, with the breakdown in classroom discipline, bullying and social withdrawal have become social problems in recent years; there is an ever-increasing expectation toward physical education to play the role of developing proper social attitudes as well cultivating morality (Hellison, 2003; Otsu et al., 2010; Umegaki \& Tomozoe, 2010; Tomozoe, 2009).

Helping behavior is one of the prosocial behaviors expected to be developed by school physical education. Although the definition of helping behavior varies slightly among researchers, it is commonly characterized by spontaneous activities as well as by the provision of benefits to others (Nagai, 2011). Recently, the importance of helping behavior has been noted in research fields other than psychology. For instance, based on many years of primatology research, Yamagiwa (2012) noted that against the backdrop of change in the role of the family and community, one's efforts to work together with his/her peers to complement one another towards a single goal and improve one's ability, would lead to maintenance of society and happiness. Empathy towards situations faced by others and behavior for helping others are important in modern society which sees individualism advancement and must be encouraged in schools. Some studies have already been carried out, with a focus on a game of tag, as child's play, to promote children's helping behavior through participation in the game (Ueno, 2014, 2015).

The game of tag is typically carried out according to rules that encourage players to escape from "it" by avoiding being tagged for as long as possible. However, some variants of tag have the rule that encourages players to not only escape from it, but also help peers who have been caught by it or those that are also escaping from it. After developing a tag variant, Nakama-oni (helping tag), in which these helping behaviors are frequently seen, Ueno (2014) examined 24 elementary school children in the second or third grade who participated in this tag game. Results reported a raised possibility of enhancing helping behavior-related self-efficacy through participation in tag, which anticipates children's ability to help their teachers or peers.

Regarding the process of how one's helping experience generates subsequent helping behavior, Midlarsky (1991) proposed a model in which the emotions or feelings associated with assistance achievement, such as meaningfulness of life, heightened self-efficacy, and a positive mood, produced as a result of children's helping behavior, leads to elevated motivation towards helping behavior and subsequent helping behavior. Based on this model, Ueno $(2014,2015)$ defined "helping behavior-related self-efficacy" as an expectation of efficacy towards helping behavior and used children's helping behavior-related self-efficacy in their school life as a variable for predicting another helping behavior. According to Ueno (2015), the frequency of helping behavior performed in a Nakama-oni has not been examined and the validity of Nakama-oni as an activity for experiencing helping behavior has not been revealed. Further, it is unclear whether the same result can be obtained during physical education. Therefore, it is necessary to further evaluate the frequency of helping behavior experienced in $\mathrm{Na}$ - 
kama-oni through repeated practices and to assess the effect of participation in Nakama-oni on children's helping behavior-related self-efficacy.

Thus, this study aims to reveal frequency of helping behavior experienced in Nakama-oni, developed by Ueno $(2014,2015)$, by adopting it in physical education, as well as the effect of participating in Nakama-oni on children's helping behavior-related self-efficacy. Bandura (1977) argues that efficacy expectation can be generalized beyond the specific situation. If helping behavior-related self-efficacy can be enhanced through physical education and be generalized into school life, it may promote helping behavior of children not only during physical education, but also in their school life.

\section{Method}

\subsection{Participants}

Sixty children ( 33 males, 27 females, ten to eleven years old) in the fifth grade at an elementary school participated in this study. The participants were informed that their participation in this study was voluntary and confidential, and that they would not be penalized in any way if they chose not to participate. The principal and homeroom teachers of the school were provided an explanation regarding the purpose of this study before their cooperation was requested. This study was carried out following ethical guidelines of the Japanese society of sport psychology after being approved by the research board of the institute to which the author belongs.

\subsection{Procedure}

Participants belonged to one of two classes. Both classes played one round of two types of tag, "Nakama-oni" and "regular tag", in reverse order in a physical education class. The first tag lessons for both classes took place successively in the same day, followed by the second tag lessons occurring successively after a 2-day interval. Each lesson was 45 minutes long and all lessons were taught by the same instructor.

In both tag games, children were divided into groups of 6 to 7 members, and each group played tag for about 15 minutes. Children were given approximately 5 minutes to recollect, mainly based on questions regarding the "state of mind during escape and the manner of escape". Subsequently, they played the same tag game for about 15 minutes, followed by recollection for roughly 5 minutes. The 15-minute tag activity consisted of six rounds of tag with a minute of intermission between each round. In each round, the role of it was replaced among children in a group.

\subsection{Game Rules}

1) Regular tag. This tag game was conducted within a field approximately half the size of a volleyball court. Before the start of the game, participants received an explanation of the game's rules in which they were encouraged to run away 
from it for as long as possible. Escapers were equipped with two tags around their waist. With the starting sign given by the instructor, the escapers ran around the field to avoid their tags from being snatched by it for around a minute until there was the closing signal by the instructor. Children who had both tags snatched were moved out of the field.

2) Nakama-oni. The same groups who played the regular tag game, played Nakama-oni within the same space. Before the start of the game, participants received an explanation of the rules in which they were encouraged to "run away from it for as long as possible while helping others". Five escapers ran around the field to avoid being tagged by it. If the escapers held hands with each other, it could not tag them. In contrast, the escapers were allowed to hold hands with only one child at a time. When an escaper was tagged by it, the escaper was brought to the original position, and the chase started again with the signal of it. Since Nakama-oni must be played by 6 players, in groups of 7 members, 1 member did not participate in the game alternatively for each game.

\subsection{Measures}

1) Frequency of helping behavior and experience of being helped by others. The rule of Nakama-oni is characterized by the availability granted to escapers to help peers from it. Thus, the survey included two questions which were asked after the implementation of tag to assess the frequency of helping behavior together with the frequency of the experience of being helped by others: (1) "how much were you able to help your peers?"; and (2) "how much did your peers help you?" For each of question, a 4-point scale ranging from (1) could not help them at all ("they did not help me at all") to (4) helped them all the time ("they helped me all the time") was used for assessment, and the scores obtained from each item were used in the analysis.

2) Helping behavior-related self-efficacy scale. Ueno (2017) developed the helping behavior-related self-efficacy scale for 10 to 12 -year old children, which assesses children's self-efficacy towards helping behavior in their school life. The scale consists of 1 factor with 6 items such as "help the teacher to carry the luggage" and "encourage peers who were disappointed due to failure". A 6-point scale ranging from (1) "I do not think I can do it at all" to (6) "I am certain I can do it", was used in assessing each item. The mean value for all items was used for analysis. The higher score of this scale means helping behavior-related self-efficacy were better. Participants completed this scale before and after each lesson.

\section{Results}

\subsection{Differences in Helping Behavior and Experience of Being Helped by Others between Two Types of Tag Games}

The frequencies of helping behavior and experience of being helped by others during the two tag games were compared. A t-test showed that the difference in 
mean values between the two tag games was significant (Helping behavior: $t(59)$ $=6.42, p<0.001, d=0.79$, Experience of being helped by others: $t(59)=6.34, p$ $<0.001, d=0.97$ (Table 1)).

Both the frequencies of helping behavior and experience of being helped by others were greater when playing Nakama-oni than when playing regular tag.

\subsection{The Effect of Participating in Nakama-oni on Children's Helping Behavior-Related Self-Efficacy}

The type of tag game (Nakama-oni or regular tag) and the survey timing (before and after implementation) were selected as the independent variables and helping behavior-related self-efficacy was selected as the dependent variable. A two-way repeated measure ANOVA was performed with these variables. As a result, the interaction between the two independent variables was significant $\left(F(1,59)=9.29, p<0.01, \eta^{2}=0.15\right.$, Table 2 , Table 3$)$. Simple main effects were examined and results showed that the difference in mean values before and after implementation of Nakama-oni was significant because the value was higher after implementation $\left(F(1,118)=6.43, p<0.05, \eta^{2}=0.05\right)$. The difference in mean values after implementation of Nakama-oni and regular tag was significant since the value was higher after Nakama-oni rather than after regular tag $(F(1,118)=$ 9.35, $\left.p<0.01, \eta^{2}=0.07\right)$. These results suggest that participating in Nakama-oni enhances children's helping behavior-related self-efficacy.

\section{Discussion}

\subsection{Frequencies of Helping Behavior and Experience of Being Helped by Others While Playing Nakama-oni}

The two-way ANOVA showed that the frequencies for both helping behavior and experience of being helped by others were higher in Nakama-oni than in regular tag. These results demonstrate that Nakama-oni is a tag game where both helping behavior and experience of being helped by others can be seen.

Based on the model proposed by Midlarsky (1991), it is supposed that the first step toward creating situations where helping behavior is frequently exhibited is for individuals to have the experience of helping others. It is believed that self-efficacy or positive mood experienced in children resulting from helping behavior, enhances their motivation for further helping behavior. Generally, people do not often encounter opportunities to help others in everyday life.

Table 1. Differences in helping behavior and experience of being helped by others between two types of tag games.

\begin{tabular}{|c|c|c|c|c|c|c|c|}
\hline & \multicolumn{2}{|c|}{ Nakama-oni } & \multicolumn{2}{|c|}{ Regular tag } & \multirow{2}{*}{$t$} & \multirow{2}{*}{$t$} & \multirow{2}{*}{$d$} \\
\hline & Mean & $S D$ & Mean & $S D$ & & & \\
\hline Helping behavior & 2.75 & 0.80 & 2.03 & 1.01 & 6.42 & $* * *$ & 0.79 \\
\hline Experience of being helped by others & 2.75 & 0.82 & 1.90 & 0.93 & 6.34 & $* * *$ & 0.97 \\
\hline
\end{tabular}

Note: $d f=59 ; S D=$ standard deviation; ${ }^{* *} p<0.001$ (two-tailed test). 
Table 2. Descriptive statistics at pre and post tag game on children's helping behavior-related self-efficacy.

\begin{tabular}{cccccc}
\hline \multirow{2}{*}{} & & \multicolumn{2}{c}{ Pre } & \multicolumn{2}{c}{ Post } \\
\cline { 3 - 6 } & & Mean & $S D$ & Mean & $S D$ \\
\hline $\begin{array}{c}\text { Helping behavior-related } \\
\text { self-efficacy }\end{array}$ & Nakama-oni & 4.10 & 1.32 & 4.33 & 1.30 \\
& Regular tag & 4.19 & 1.30 & 4.05 & 1.46 \\
\hline
\end{tabular}

Note: $S D=$ standard deviation

Table 3. Results of the two-way repeated measure anova on children's helping behavior-related self-efficacy.

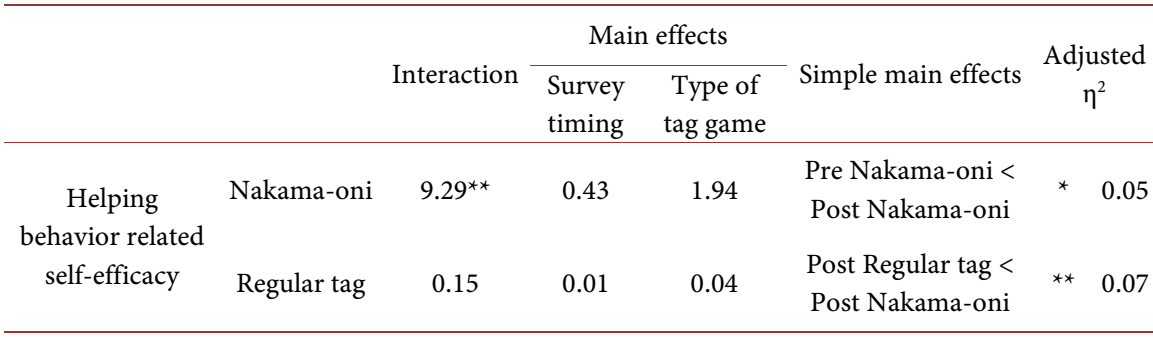

Note: $F$ value was shown at the first line of the interaction and main effects. Adjusted $\eta^{2}$ was shown at the second line of the interaction and main effects. ${ }^{*} p<0.01,{ }^{*} p<0.05$.

Further, in school education, it is not easy to create situations where every student can learn helping behavior.

Since Nakama-oni is a game, it allows children to repeatedly experience helping behavior under conditions governed by rules. The helping behavior can be recognized by peers, even if it fails to help them, and it can be tried a number of times. Despite the fact that it is a game, Nakama-oni may provide opportunities for children to experience helping behavior more often, and so may represent a first step toward creating situations where helping behavior is frequently exhibited by children.

In addition, it was found that Nakama-oni provided opportunities for children to not only perform helping behavior, but also experience help from others. Matsui and Ura (1998) proposed a model in which retributive behavior is performed as a retribution for assistance provided by others or as compensation for damage inflicted on them while providing assistance. In Nakama-oni, the range children are allowed to run is limited and there is no need for tags. Therefore, it is necessary for children, even if they are fast runners, to receive help from their peers in order to avoid being caught by it. In this study, when children received help from their peers, a sense of gratitude for their efforts to help and for running the risk of being caught in delivering assistance was generated. Further, it was found that the experience of being helped by others while playing Nakama-oni may have enhanced children's motivation for further helping behavior.

\subsection{Improvement in Children's Helping Behavior-Related Self-Efficacy through Participation in Nakama-oni}

The ANOVA results showed that participation in Nakama-oni enhanced child- 
ren's helping behavior-related self-efficacy. The higher frequency of helping behavior in Nakama-oni compared to that in regular tag suggested that children's helping behavior-related self-efficacy was enhanced through their experience of helping others or being helped by others during the game of Nakama-oni.

One of the factors influencing the improvement of self-efficacy is the "performance accomplishment" (Bandura, 1977). In a game of Nakama-oni, most children have an experience of helping peers in the same group. In order to help a peer who is chased by it and is in need of help, somebody who is his or her neighbor must take necessary action. However, carelessly moving closer to the individual in need, would increase the risk of being caught by it. Results suggest that children's experience of having been able to spontaneously act when a peer was in trouble, gives him/her confidence and heightens their sense of helping behavior-related self-efficacy, anticipating their ability to help their peers in their school life.

According to the model proposed by Midlarsky (1991), the emotions or feelings associated with assistance outcomes, such as meaningfulness of life and heightened self-efficacy produced from children's helping behavior, lead to elevated motivation towards helping behavior; and hence, to subsequent helping behavior. According to this model, self-efficacy in helping others is not directly associated with helping behavior, since influence from other factors, such as motivation towards helping behavior, is assumed until it is performed as a helping behavior. However, Ueno (2017) revealed that there was a correlation of approximately 0.58 between helping behavior-related self-efficacy and past experience of helping others, suggesting that helping behavior-related self-efficacy is a significant factor explaining actual helping behavior. These findings support the assumption that enhanced helping behavior-related self-efficacy through the participation in Nakama-oni may promote helping behavior in school life.

\section{Conclusion}

In this study, children played Nakama-oni (Ueno, 2014, 2015), a tag game promoting helping behavior in a physical education class. In doing so, the frequency of helping behavior in Nakama-oni, together with the effect of participation in Nakama-oni in a physical education class on children's self-efficacy in helping behavior was examined.

Sixty children in the fifth grade played Nakama-oni and regular tag in a physical education class. Results showed that: 1) children experienced both helping behavior and experience of being helped by others more frequently during $\mathrm{Na}$ kama-oni, compared to regular tag; and 2) participating in Nakama-oni enhanced children's helping behavior-related self-efficacy. In conclusion, these results concur with the assumption that playing Nakama-oni, which promotes helping behavior during a physical education class, can enhance children's helping behavior-related self-efficacy.

Results of this study suggested the need to reveal in the future the extent to 
which helping behavior-related self-efficacy elevated through participation in a physical education class promotes helping behavior in actual school life. In particular, the possibility of increasing the helping behavior frequency during the class immediately after the physical education class designed to promote helping behavior is one of the topics that need to be discussed in the future.

\section{Funding}

This work was supported by Japan Society for the Promotion of Science (Grant No. 16K01622).

\section{References}

Bandura, A. (1977). Self-Efficacy: Toward a Unifying Theory of Behavioral Change. Psychological Review, 84, 191-215. https://doi.org/10.1037/0033-295X.84.2.191

Hellison, D. (2003). Teaching Responsibility through Physical Activity (2nd ed.). Human Kinetics, IL: Champaign.

Matsui, Y., \& Ura, M. (1998). Psychology in Helping Others. [人を支える心の科学.] Tokyo: SeishinShobo.

Midlarsky, E. (1991). Helping as Coping. In M. S. Clark (Ed.), Prosocial Behavior (pp. 238-264). Sage: Newbury Park.

Nagai, A. (2011). The Progress and Issues in Studies of Helping-Behavior. Chuo University Bulletin of Graduate Studies, 40, 53-69.

Otsu, A., Hosogoe, J., \& Takahashi, T. (2010). A study on Children's Cognitive Transfiguration toward Social Behavior in Physical Education Classes. Japanese Journal of Sport Education Studies, 29, 17-32. https://doi.org/10.7219/jjses.29.2_17

Tomozoe, H. (2009). Character Building in Physical Education. Tokyo: Taishukan Publishing.

Ueno, K. (2014). Effects of Participation in the Game of Tag with a Focus on Helping Others on Self-Efficacy in Helping Behavior of Children. Tottori University Education Center Bulletin, 11, 75-84.

Ueno, K. (2015). Using a Unique Tag Game to Improve Self-Efficacy in Helping Behavior of Children. In Proceedings of the 14th European Congress of Sport Psychology ( $p$. 414), Switzerland.

Ueno, K. (2017). The Impact of Participation in a Game of Tag, an Activity Promoting Helping Behavior, on Helping Behavior-Related Self-Efficacy and Subjective Adjustment to the Class Room among Children. In Proceedings of the Japanese Society of Sport Psychology 44th Annual Conference Proceedings (pp. 134-135), Japan.

Umegaki, A., \& Tomozoe, H. (2010). Research Trends on Moral Learning and Responsibility Learning in Physical Education in Articles of JTPE. Japanese Journal of Sport Education Studies, 29, 1-16. https://doi.org/10.7219/jjses.29.2_1

Yamagiwa, J. (2012). Evolutionary History of Human Family. Tokyo: University of Tokyo Press. 